\title{
Utilização da hipodermóclise por profissionais de saúde: Scoping review
}

\author{
Use of hypodermoclysis by health professionals: Scoping review \\ Uso de hipodermoclisis por profesionales de la salud: Revisión de escopo
}

Recebido: 17/07/2021 | Revisado: 21/07/2021 | Aceito: 22/07/2021 | Publicado: 30/07/2021

\author{
Síntique Sara Silva Santos \\ ORCID: https://orcid.org/0000-0002-2820-5118 \\ Universidade Federal de Alfenas, Brasil \\ E-mail: sintique.santos@sou.unifal-mg.edu.br \\ Joice Marques Ribeiro \\ ORCID: https://orcid.org/0000-0001-5292-3753 \\ Universidade Federal de Alfenas, Brasil \\ E-mail: joice.ribeiro@ sou.unifal-mg.edu.br \\ Hertz Bezerra Alves \\ ORCID: https://orcid.org/0000-0002-8411-0287 \\ Prefeitura Municipal de Belo Horizonte, Brasil \\ E-mail: hertzbezerra@gamil.com \\ Andréia Cristina Barbosa Costa \\ ORCID: https://orcid.org/0000-0003-3484-9638 \\ Universidade Federal de Alfenas, Brasil \\ E-mail: andreia.barbosa@unifal-mg.edu.br \\ Adriana Olimpia Barbosa Felipe \\ ORCID: https://orcid.org/0000-0003-4491-5750 \\ Universidade Federal de Alfenas, Brasil \\ E-mail: adriana.felipe@ unifal-mg.edu.br \\ Isabelle Cristinne Pinto Costa \\ ORCID: https://orcid.org/0000-0002-2611-8643 \\ Universidade Federal de Alfenas, Brasil \\ E-mail: isabelle.costa@unifal-mg.edu.br
}

\begin{abstract}
Resumo
Introdução: A hipodermóclise, também denominada de terapia subcutânea, é uma via de infusão alternativa, a qual visa à administração de soluções e fármacos. Essa via apresenta a mesma eficácia da endovenosa, com a vantagem de ser menos dolorosa e com raros eventos adversos. No entanto, ainda é um procedimento pouco implementado e discutido por profissionais de saúde. Objetivo: Mapear as produções científicas acerca da utilização da hipodermóclise por profissionais da saúde. Metodologia: Trata-se de uma scoping review, norteada pelo instrumento PRISMA Extension for Scoping Reviews. As buscas foram realizadas em cinco bases de dados. Os resultados foram apresentados de forma descritiva, com apresentação de gráficos e tabelas. Resultados: Foram incluídos 27 estudos, sendo a maioria desenvolvido por enfermeiros brasileiros publicados nos anos de 2016 e 2019, no idioma português, que buscaram avaliar as principais indicações e reações adversas acerca da hipodermóclise. Conclusões: Esta scoping review oferece evidências do protagonismo da enfermagem na realização da hipodermóclise e do avanço do conhecimento da referida área. Além de compilar informações que podem direcionar os profissionais de saúde para a utilização da referida técnica, com vistas a garantir o sucesso da terapia e a segurança do paciente. Palavras-chave: Hipodermóclise; Terapia subcutânea; Profissional de saúde.
\end{abstract}

\begin{abstract}
Introduction: Hypodermoclysis, also called subcutaneous therapy, is an alternative infusion route, which aims to administer solutions and drugs. This route has the same efficacy as the intravenous route, with the advantage of being less painful and with rare adverse events. However, it is still a procedure little implemented and discussed by health professionals. Objective: To map scientific productions on the use of hypodermoclysis by health professionals. Methodology: This is a scoping review, guided by the PRISMA Extension for Scoping Reviews instrument. Searches were performed in five databases. The results were presented descriptively, with the presentation of graphs and tables. Results: Twenty-seven studies were included, most of them developed by Brazilian nurses, published in 2016 and 2019, in Portuguese, which sought to assess the main indications and adverse reactions regarding hypodermoclysis. Conclusions: This scoping review offers evidence of the role of nursing in the performance of hypodermoclysis and the advancement of knowledge in this area. In addition to compiling information that can guide health professionals to use this technique, in order to ensure the success of therapy and patient safety.
\end{abstract}

Keywords: Hypodermoclysis; Subcutaneous therapy; Healthcare professional.

\section{Resumen}

Introducción: La hipodermoclisis, también llamada terapia subcutánea, es una vía de infusión alternativa, que tiene como objetivo la administración de soluciones y fármacos. Esta vía tiene la misma eficacia que la vía intravenosa, con la ventaja de 
ser menos dolorosa y con raros eventos adversos. Sin embargo, todavía es un procedimiento poco implementado y discutido por los profesionales de la salud. Objetivo: Mapear producciones científicas sobre el uso de hipodermoclisis por profesionales de la salud. Metodología: Esta es una revisión de alcance, guiada por el instrumento PRISMA Extension for Scoping Reviews. Las búsquedas se realizaron en cinco bases de datos. Los resultados se presentaron de forma descriptiva, con la presentación de gráficos y tablas. Resultados: Se incluyeron 27 estudios, la mayoría de ellos desarrollados por enfermeras brasileñas, publicados en 2016 y 2019, en portugués, que buscaban evaluar las principales indicaciones y reacciones adversas de la hipodermoclisis. Conclusiones: Esta revisión de alcance ofrece evidencia del papel de la enfermería en la realización de la hipodermoclisis y el avance del conocimiento en esta área. Además de recopilar información que pueda orientar a los profesionales de la salud en el uso de esta técnica, con el fin de garantizar el éxito de la terapia y la seguridad del paciente.

Palabras clave: Hipodermoclisis; Terapia subcutánea; Profesional de la salud.

\section{Introdução}

A hipodermóclise, também conhecida como terapia subcutânea, é considerada uma prática antiga que teve seu primeiro relato em 1913, no entanto, por conta dos eventos adversos decorridos de sua utilização inadequada, como o uso de soluções hipertônicas, a prática passou a ser inutilizada (Zironde et al., 2014; Bruno, 2015;). Tal técnica é realizada com vistas a administrar soluções na hipoderme, camada mais profunda da pele, cuja vascularização é similar à que se observa nos músculos (Pontalti et al., 2018).

É oportuno assinalar que a referida técnica vem sendo utilizada em diversas instituições de saúde, a nível internacional e nacional, como um valioso recurso para pacientes que não possuem a possibilidade da utilização da via oral por problemas com a deglutição ou da via endovenosa, na qual a fragilidade das veias e a perda de elasticidade não permitem a punção do acesso venoso, além de permitir maior conforto, praticidade e menores riscos quando comparada a um cateter venoso central (Vidal et al., 2015). É descrita também como uma prática simples de ser realizada e mais barata que as demais técnicas (D'Aquino \& Souza, 2012).

Porquanto, a referida via tem suas vantagens, benefícios, indicações e poucas complicações possíveis, podendo contribuir para a promoção da qualidade de vida do paciente. Para Lopes (2014) às imensas vantagens da terapia subcutânea superam as suas possíveis desvantagens.

Sob esse prisma, a hipodermóclise é considerada uma excelente via alternativa para pacientes que estão em cuidados paliativos, como também em extremos de idade, uma vez que esses grupos, podem apresentar maiores fragilidades com a via endovenosa. Portanto, a via subcutânea é de fácil acesso, de eficácia comprovada, pouco agressiva e com poucos efeitos secundários, bem como, confortável para o cliente (Gomes et al., 2017).

Ressalta-se ainda, que atualmente a dificuldade no uso da técnica se dá principalmente pela falta de documentações padronizadas, que guiem a aplicação da mesma, orientando com relação a quais medicações podem ser utilizadas, diluições e compatibilidades entre elas, podendo ocasionar insegurança no uso por parte dos profissionais da saúde (Nunes \& Souza, 2016).

Assim, considerando a relevância dessa temática, é de suma importância que os profissionais de saúde conheçam sobre o assunto, uma vez que possibilitará refletir sobre a importância de seu papel ao utilizar esse método como recurso terapêutico. Adicionalmente, tais conhecimentos podem minimizar traumas mecânicos e tissulares, promover conforto, diminuir o estresse e dor por punções repetidas e sem êxito para a infusão de fluidos e medicamentos, além de minimizar consideravelmente o risco de infecção.

Diante de tais ponderações e pelo fato de existirem poucas informações na literatura sobre este assunto, surgiu o interesse de analisar e compilar, por meio de uma revisão de escopo, o que as literaturas nacionais e internacionais disponibilizam sobre a utilização da hipodermóclise por profissionais de saúde. 


\section{Metodologia}

O estudo elaborado é uma revisão de escopo, scoping review. De maneira geral, a scoping review compreende um tipo de revisão de literatura que tem como técnica "mapear" estudos relevantes no campo de interesse. As análises de escopo são comumente usadas para reconhecimento e para definições de limites conceituais a respeito de um tópico ou campo. Essas são, portanto, de particular utilidade quando um corpo de literatura ainda não foi amplamente revisto, ou exibe uma natureza complexa e ou heterogênea (Ring et al., 2012), como é o caso da hipodermóclise.

Esta revisão de escopo foi conduzida segundo as recomendações do Joanna Briggs Institute Reviewer's Manual, a partir das seguintes etapas:1. identificar a questão de pesquisa, 2. identificar estudos relevantes, 3. seleção de estudo, 4. mapear os dados e 5. comparar, sumarizar e descrever os resultados (Peters et al., 2020). O relato desta revisão seguiu o proposto pelo PRISMA extension for scoping reviews (PRISMA-ScR), que é dividido em sete domínios e 22 itens, que dispõem de recomendações acerca do título, do resumo, da introdução, do método, do resultado, da discussão, da conclusão e do financiamento do estudo (Tricco et al., 2018).

\section{Identificação da questão de pesquisa}

O estudo baseia-se na estratégia mnemônica População, Conteúdo e Contexto (PCC), utilizando os profissionais de saúde como população, a hipodermóclise como conteúdo, e o contexto foi o quantitativo de estudos, as bases em que foram publicados, o idioma e a frequência de publicações nos últimos 10 anos. A escolha por esse intervalo de tempo se deu devido a atualidade da literatura levantada. Assim, ajustando-se os tópicos-chave do PCC ao objetivo do estudo, gerou-se a seguinte questão norteadora: Qual a caracterização das publicações sobre a utilização da hipodermóclise por profissionais de saúde?

\section{Identificação de estudos relevantes}

Uma busca preliminar foi realizada na base de dados Pubmed (US National Library of Medicine) para determinar as palavras-chave e descritores mais utilizados para a indexação de estudos relacionados ao tema de interesse desta revisão.

A partir da questão de pesquisa e dessa busca preliminar, foram selecionados Descritores controlados em Ciências da Saúde (DeCS) e Medical Subject Headings (MeSH), em português, inglês e espanhol: 'Hipodermóclise'; 'Hypodermoclysis'; 'Hipodermoclisis".

Destaca-se que a busca nas bases de dados ocorreu entre outubro e dezembro/2020 por dois revisores independentes, responsáveis pela triagem e identificação de estudos relevantes nas seguintes bases: Sistema Online de Busca e Análise de Literatura Médica - MEDLINE® (acesso via PubMed); Scopus; Cumulative Index to Nursing and Allied Health Literature (CINAHL); Web of Science (WoS) e Latino-Americana de Informação Bibliografia (LILACS).

Após a determinação dos descritores e a criação da estratégia acima, procedeu-se à realização das buscas em cada biblioteca e/ou base de dados. O acesso ocorreu por meio do Portal de Periódicos CAPES, com o uso da plataforma CAFe (Comunidade Acadêmica Federada), serviço que facilita a disponibilização e o acesso a benefícios digitais por meio do login utilizado para a respectiva universidade cadastrada.

\section{Seleção dos estudos}

Durante a seleção das publicações, foram utilizados os seguintes critérios de inclusão: 1) artigos científicos publicados em inglês, português ou espanhol, no período de 2010 a 2019, cujos títulos contemplem o termo 'hipodermóclise' ou "via subcutânea" e que estejam disponibilizados na íntegra para acesso online; 2) o estudo ser realizado com humanos. Foram excluídos estudos duplicados, capítulos de livros, normas, protocolos e resumos publicados em anais. 
O processo de seleção dos estudos deu-se a partir da leitura criteriosa dos títulos e resumos dos estudos obtidos nas fontes de informação, sendo excluídos aqueles que não apresentavam os elementos do conceito e os duplicados. Em seguida, realizou-se a análise do texto completo e foram elencados todos que, de alguma forma, apresentavam a utilização da via subcutânea por profissionais de saúde. Quaisquer divergências foram resolvidas por consenso e pela decisão de um terceiro revisor.

Os resultados das buscas foram exportados para o gerenciador de referências denominado EndNote ${ }^{\circledR}$, por meio do qual ocorreu a organização de referências identificadas em diferentes bases de dados eletrônicas e remoção de estudos duplicados.

\section{Extração de dados}

Durante a extração dos dados, utilizou-se o formulário recomendado pelo Instituto Joanna Briggs (JBI) (Peters et al., 2020) com o intuito de facilitar a síntese de informações e a qualidade das recomendações, que elenca dados importantes na consecução de uma revisão: a) autor(es); b) ano de publicação; c) localização do estudo; d) população do estudo e tamanho da amostra (quando aplicável); e) tipo de intervenção (se aplicável); f) objetivos do estudo; g) Metodologia/ Métodos; h) resultados importantes; i) agência financiadora.

Destaca-se que os pesquisadores desse estudo realizaram uma adaptação do referido protocolo, acrescentando ao mesmo a extração dos seguintes dados: título do artigo; idioma; ano de publicação; metodologia; formação do primeiro autor; amostra; objetivo; efeitos adversos, indicação e resultados principais.

\section{Sumarização e análise dos dados}

Procedeu-se uma análise sobre a extensão, natureza e distribuição geral dos estudos incluídos, realizando-se uma identificação de temas recorrentes para então, realizar uma abordagem mais específica e consistente dos dados de acordo com a questão norteadora desta revisão. Posteriormente, os estudos foram comparados quanto aos achados comuns e recorrentes em relação à hipodermóclise para o estabelecimento de semelhanças e diferenças (Levac, Colquhoun \& O’Brien, 2010).

Por conseguinte, foi realizada uma análise descritiva dos dados empregando frequências relativas e absolutas, bem como a caracterização e apresentação dos resultados em figuras, gráficos e tabelas. Por não envolver seres humanos, este estudo não foi submetido à aprovação do Comitê de Ética em Pesquisa. Por não ser de natureza experimental, apresenta risco mínimo. Ressalta-se que os preceitos da Lei n. ${ }^{\circ}$ 9.610/98 foram integralmente cumpridos com vistas a se preservar e respeitar as ideias, os conceitos e as definições dos autores dos estudos primários selecionados.

\section{Resultados}

A amostra desta revisão foi composta por 27 estudos. O processo de seleção e inclusão dos artigos está apresentado na figura 1. 
Figura 1 - Processo de identificação e inclusão dos estudos - Preferred Reporting Items for Systematic re- views and MetaAnalyses extension for Scoping Reviews (PRISMA-ScR) Checklist, Alfenas, MG, Brasil, 2020.

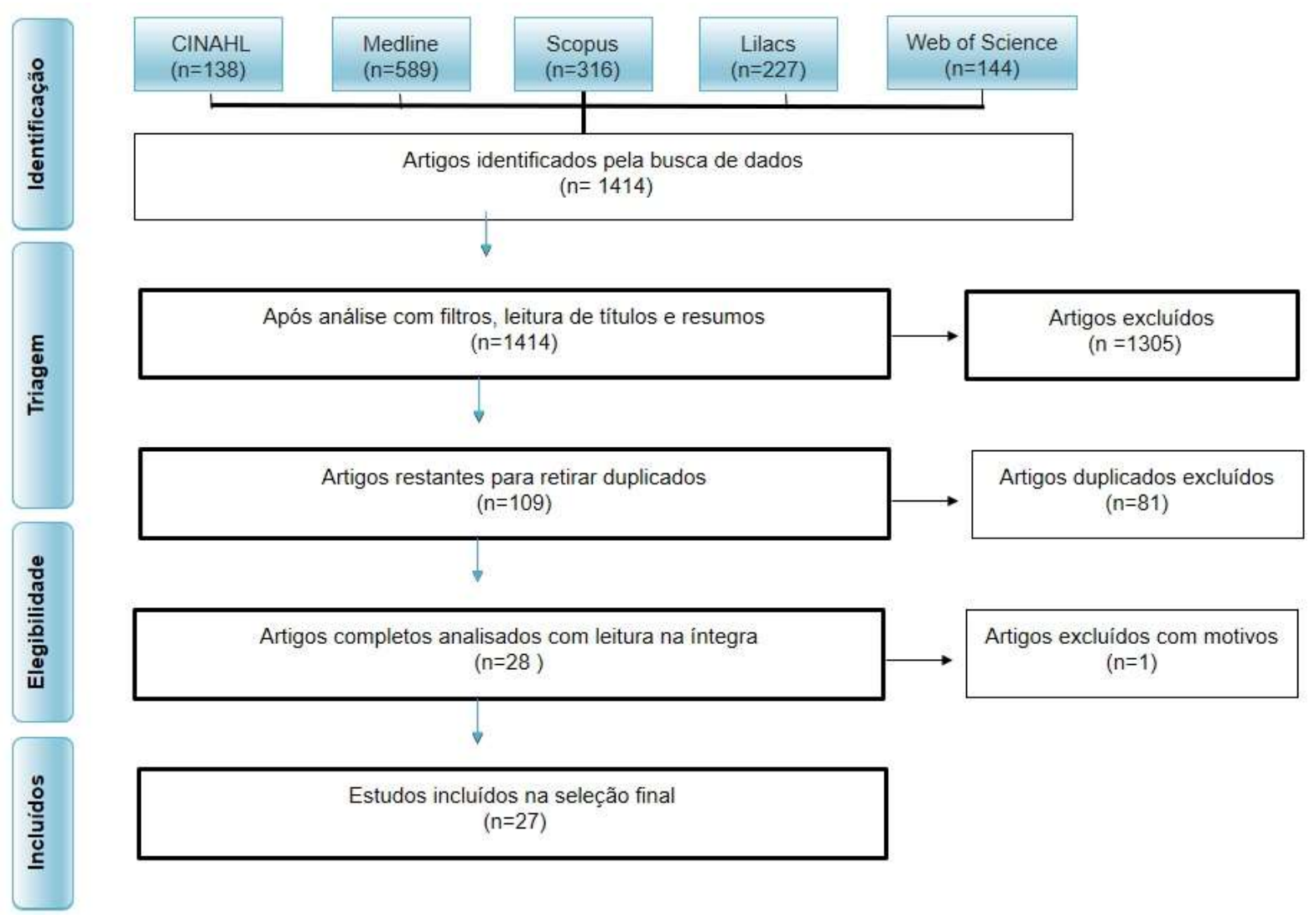

Fonte: Dados da Pesquisa (2020).

O Gráfico 1, apresenta o número de publicações acerca do tema hipodermóclise no intervalo de tempo selecionado para este estudo. É possível observar que os estudos sobre a referida técnica demonstrou-se constante e com um salto no ano de 2016, com quatro publicações e no ano de 2019 com seis publicações.

Gráfico 1 - Disseminação das publicações inseridas no estudo no intervalo de 2010 a 2019, Alfenas, Minas Gerais, Brasil, 2020. $(n=27)$

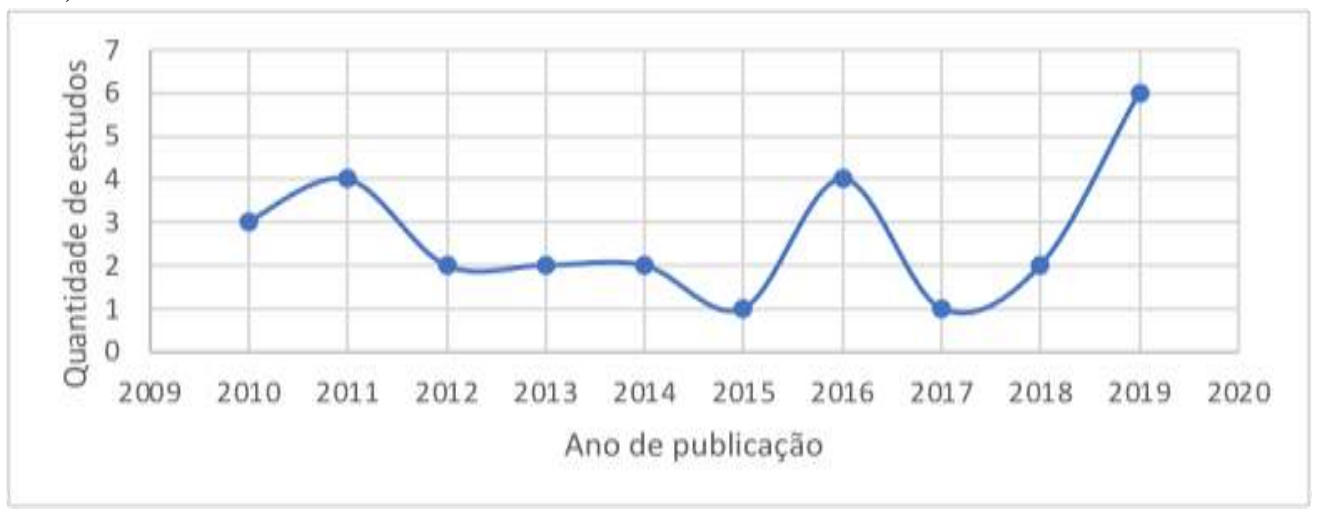

Fonte: Dados da Pesquisa (2020).

A Tabela 1, apresenta dados relacionados ao idioma de publicação, à formação profissional dos pesquisadores, às metodologias e os países de publicação. Em relação ao idioma de publicação da produção científica da amostra deste estudo, 
observa-se que a língua portuguesa foi a predominante, com 14 (52\%) trabalhos, seguidos pelas línguas inglesa, com dez (37\%) e espanhola, com três (11\%). Quanto à formação dos primeiros autores identificados na ordem numérica da lista de autores das publicações, verificou-se que os profissionais que mais publicaram sobre o tema foram os enfermeiros, correspondendo a 19 (70\%), seguidos de médicos, com seis (22\%) e farmacêuticos, com dois (7\%). No que se refere a escolha das metodologias utilizadas nas pesquisas, destacam-se que as revisões foram predominantes, sendo empregadas por 13 (48\%) estudos, seguidas por estudos qualitativos prospectivos, com sete (26\%), estudos de caso, com três (11\%), relatos de experiência, com três (11\%) e um estudo metodológico (4\%). Os países que mais publicaram foram o Brasil (51\%), Estados Unidos (22\%) e Canadá (7\%), os demais publicaram apenas um estudo para cada.

Tabela 1 - Resultados relacionados ao idioma, países de publicação, área de formação dos autores principais e metodologias adotadas nos estudos, Alfenas, Minas Gerais, Brasil, 2020. ( $\mathrm{n}=27)$

\begin{tabular}{ccc}
\hline Variáveis & Número de Estudos & Porcentagem $(\%)$ \\
Idiomas & 14 & $52 \%$ \\
Português & 10 & $37 \%$ \\
Inglês & 3 & $11 \%$ \\
Espanhol & 27 & $100 \%$ \\
Total & & \\
Formação do autor principal & 19 & $70 \%$ \\
Enfermagem & 6 & $22 \%$ \\
Medicina & 2 & $7 \%$ \\
Farmácia & 27 & $100 \%$ \\
Total & & \\
Metodologia & 13 & $48 \%$ \\
Revisão & 7 & $26 \%$ \\
Qualitativo prospectivo & 3 & $11 \%$ \\
Relato de experiência & 3 & $11 \%$ \\
Relato de caso & 1 & $4 \%$ \\
Metodológica & 27 & $100 \%$ \\
Total & & \\
Países & 14 & $51 \%$ \\
Brasil & 6 & $22 \%$ \\
Estados Unidos & 2 & $7 \%$ \\
Canadá & 1 & $4 \%$ \\
Inglaterra & 1 & $4 \%$ \\
Reino Unido & 1 & $4 \%$ \\
Peru & 1 & $4 \%$ \\
Colômbia & 1 & $4 \%$ \\
Cuba & 27 & $100 \%$ \\
Total & & \\
\hline & & \\
\hline
\end{tabular}

Fonte: Dados da Pesquisa (2020).

O quadro 1 apresenta a caracterização dos estudos pertencentes à amostra final, considerando o objetivo, indicações, população e/ou amostra do estudo, reações adversas e principais conclusões, segundo o Joanna Briggs Institute. 
Quadro 1 - Caracterização dos estudos quanto ao objetivo, às indicações da hipodermóclise, à população e/ou amostra, às reações adversas e às principais conclusões. Alfenas, MG, Brasil, 2020.

\begin{tabular}{|c|c|c|c|c|c|}
\hline Artigo & Objetivo & Indicações & $\begin{array}{l}\text { Participa } \\
\text { ntes ou } \\
\text { amostra }\end{array}$ & $\begin{array}{l}\text { Reações } \\
\text { Adversas }\end{array}$ & Principais conclusões \\
\hline $\begin{array}{l}\text { Hydrating Your } \\
\text { Patient the Easy Way: } \\
\text { Hypodermoclysis }\end{array}$ & $\begin{array}{l}\text { Descrever o uso da } \\
\text { hipodermóclise em uma } \\
\text { instituição de ronga } \\
\text { permanência em idosos } \\
\text { com leve ou moderada } \\
\text { desidratação }\end{array}$ & $\begin{array}{l}\text { Indicado a pacientes com } \\
\text { desidratação leve ou } \\
\text { moderada }\end{array}$ & $\begin{array}{l}\text { Residente } \\
\text { s de uma } \\
\text { Instituiçã } \\
\text { o de } \\
\text { Longa } \\
\text { Permanê } \\
\text { ncia } \\
\text { (ILP) }\end{array}$ & $\begin{array}{l}\text { Edema, } \\
\text { vermelhidão e dor } \\
\text { local, punção de } \\
\text { vasos sanguíneos } \\
\text { e raramente } \\
\text { sobrecarga de } \\
\text { fluidos. }\end{array}$ & $\begin{array}{l}\text { A hidratação subcutânea é um método } \\
\text { seguro e fácil, baseado em evidências de } \\
\text { prevenção e tratamento de desidratação } \\
\text { na população idosa. }\end{array}$ \\
\hline $\begin{array}{l}\text { Hipodermóclise: } \\
\text { revisão de literatura } \\
\text { para auxiliar a prática } \\
\text { clínica }\end{array}$ & $\begin{array}{l}\text { Analisar, na literatura, as } \\
\text { informações disponíveis } \\
\text { sobre os medicamentos que } \\
\text { podem ser administrados } \\
\text { através da hipodermóclise. }\end{array}$ & $\begin{array}{l}\text { Reidratação, dor e } \\
\text { infecção }\end{array}$ & $\begin{array}{ll}\text { Não se } \\
\text { aplica }\end{array}$ & $\begin{array}{l}\text { Dor, inflamação } \\
\text { no local da } \\
\text { punção e, até } \\
\text { mesmo, edemas e } \\
\text { necroses teciduais. }\end{array}$ & $\begin{array}{l}\text { A técnica da hipodermóclise é uma } \\
\text { metodologia segura, eficaz, barata e de } \\
\text { fácil aplicabilidade e aparenta trazer } \\
\text { alguns benefícios. }\end{array}$ \\
\hline $\begin{array}{l}\text { A multifaceted } \\
\text { intervention to address } \\
\text { a case cluster of } \\
\text { cellulitis associated } \\
\text { with hypodermoclysis } \\
\text { in a geriatric complex } \\
\text { continuing care unit }\end{array}$ & $\begin{array}{l}\text { Avaliar se uma intervenção } \\
\text { multifacetada pode reduzir } \\
\text { a incidência de celulite em } \\
\text { pacientes recebendo } \\
\text { hipodermóclise em um } \\
\text { tratamento continuado em } \\
\text { uma unidade geriátrica. }\end{array}$ & $\begin{array}{l}\text { Reduzir a incidência de } \\
\text { celulites em pacientes } \\
\text { com hipodermóclise }\end{array}$ & $\begin{array}{l}\text { Um } \\
\text { grupo de } \\
12 \\
\text { pessoas } \\
\text { com } \\
\text { celulite } \\
\text { associada } \\
\text { ao uso de } \\
\text { hipoderm } \\
\text { óclise }\end{array}$ & $\begin{array}{l}\text { Edema local, dor } \\
\text { no local da } \\
\text { infusão, } \\
\text { equimoses } \\
\text { celulite }\end{array}$ & $\begin{array}{l}\text { Mostra que a educação permanente das } \\
\text { enfermeiras nas unidades de cuidados } \\
\text { contínuos e complexos, além da } \\
\text { mudança da técnica asséptica para a } \\
\text { realização da hipodermóclise, } \\
\text { diminuíram os casos de celulite } \\
\text { associados à hipodermóclise. }\end{array}$ \\
\hline $\begin{array}{l}\text { Hypodermoclysis: An } \\
\text { alternative to I.V. } \\
\text { infusion therapy }\end{array}$ & $\begin{array}{l}\text { Descrever o } \text { uso da } \\
\text { hipodermóclise } \\
\text { hidratação de para } \\
\text { com desidratação leve a } \\
\text { moderada }\end{array}$ & $\begin{array}{l}\text { Desidratação leve a } \\
\text { moderada }\end{array}$ & $\begin{array}{l}\text { Não se } \\
\text { aplica }\end{array}$ & $\begin{array}{l}\text { Prurido, } \\
\text { queimação ou } \\
\text { vazamento no } \\
\text { local; eritema; } \\
\text { edema; } \\
\text { endurecimento; } \\
\text { sangramento; } \\
\text { infecção; reação } \\
\text { de } \\
\text { hipersensibilidade } \\
\text { a hialuronidase }\end{array}$ & $\begin{array}{l}\text { Introduz as premissas básicas para a } \\
\text { hipodermóclise através de um episódio } \\
\text { clínico pediátrico. }\end{array}$ \\
\hline $\begin{array}{l}\text { Hypodermoclysis: } \\
\text { renewed interest in an } \\
\text { old technique }\end{array}$ & $\begin{array}{l}\text { Apresentar a técnica e o } \\
\text { uso da hipodermóclise } \\
\text { como procedimento de } \\
\text { baixo custo e de fácil } \\
\text { acesso que substitui os } \\
\text { métodos intravenosos }\end{array}$ & $\begin{array}{l}\text { Desidratação leve a } \\
\text { moderada }\end{array}$ & $\begin{array}{l}\text { Não se } \\
\text { aplica }\end{array}$ & $\begin{array}{l}\text { Eritema, flebite, } \\
\text { celulite. }\end{array}$ & $\begin{array}{l}\text { Hipodermóclise entrou em desuso em } \\
1950 \text {, após erros no uso da } \\
\text { hipodermóclise, porém nas últimas } \\
\text { décadas tem voltado a entrar nos } \\
\text { protocolos dos tratamentos de longo } \\
\text { prazo devido ao seu baixo custo, } \\
\text { facilidade e mínimos efeitos colaterais. }\end{array}$ \\
\hline $\begin{array}{lr}\text { Segurança e eficácia } \\
\text { da hipodermóclise em } \\
\text { comparação com a } \\
\text { infusão de } & \text { fluido } \\
\text { intravenoso } & \text { para } \\
\text { reidratar crianças com } \\
\text { desidratação leve a } \\
\text { moderada: rro um } \\
\text { protocolo de } & \text { revisão } \\
\text { sistemática } & \end{array}$ & $\begin{array}{l}\text { Resumir as evidências } \\
\text { sobre a eficácia e } \\
\text { segurança } \\
\text { hipodermóclise (HDC) } \\
\text { para a reidratação de } \\
\text { crianças com desidratação } \\
\text { leve ou moderada }\end{array}$ & $\begin{array}{l}\text { Desidratação leve a } \\
\text { moderada }\end{array}$ & $\begin{array}{l}\text { Bebês e } \\
\text { crianças } \\
\text { de } 0 \text { dias } \\
\text { a } 10 \text { anos } \\
\text { com leve } \\
\text { ou } \\
\text { moderada } \\
\text { desidrata } \\
\text { ção }\end{array}$ & $\begin{array}{l}\text { Sinais de } \\
\text { inflamação, } \\
\text { endurecimento, } \\
\text { hematomas, } \\
\text { necrose do tecido } \\
\text { (complicação } \\
\text { tardia), } \\
\text { extravasamento, } \\
\text { sangramento, } \\
\text { prurido, abscesso, } \\
\text { febre, calafrios, } \\
\text { ansiedade e sinais } \\
\text { de sobrecarga } \\
\text { retardada } \\
\text { (taquicardia, } \\
\text { inchaço jugular, } \\
\text { hipertensão, tosse } \\
\text { e dispneia). }\end{array}$ & $\begin{array}{l}\text { Sintetiza as evidências sobre a eficácia } \\
\text { do HDC no tratamento da desidratação } \\
\text { em crianças }\end{array}$ \\
\hline $\begin{array}{l}\text { Use of } \\
\text { Hypodermoclysis to } \\
\text { manage dehydration }\end{array}$ & $\begin{array}{l}\text { Fornecer uma visão geral } \\
\text { das mudanças fisiológicas } \\
\text { que afetam a capacidade } \\
\text { dos idosos para manter um } \\
\text { equilíbrio normal de } \\
\text { fluidos se concentrando na } \\
\text { reposição de fluido } \\
\text { subcutâneo para o manejo } \\
\text { da desidratação em idosos }\end{array}$ & Desidratação & $\begin{array}{l}\text { Não se } \\
\text { aplica }\end{array}$ & $\begin{array}{l}\text { Edema local, } \\
\text { eritema e efeito } \\
\text { clínico mais lento }\end{array}$ & $\begin{array}{l}\text { Hipodermóclise é uma alternativa } \\
\text { importante para terapia IV em } \\
\text { idosos. Essa técnica tem muitas } \\
\text { vantagens, incluindo facilidade de } \\
\text { administração, menos efeitos colaterais, } \\
\text { melhor conforto do paciente, } \\
\text { administração oportuna de fluídos e } \\
\text { relação custo-eficácia, além dos custos } \\
\text { de configuração serem mínimos e o } \\
\text { potencial para redução de custos ser }\end{array}$ \\
\hline
\end{tabular}




\begin{tabular}{|c|c|c|c|c|c|}
\hline & & & & & grande. \\
\hline $\begin{array}{lr}\text { Validação } & \text { de } \\
\text { instrumento } & \text { para } \\
\text { avaliação } & \text { do } \\
\text { conhecimento } & \\
\text { profissional acerca da } \\
\text { Hipodermóclise }\end{array}$ & $\begin{array}{l}\text { Validar instrumento } \\
\text { relativo ao conhecimento } \\
\text { dos profissionais de saúde } \\
\text { em hipodermóclise }\end{array}$ & $\begin{array}{l}\text { Administração } \text { de } \\
\text { medicamentos e terapia } \\
\text { de hidratação }\end{array}$ & $\begin{array}{l}6 \\
\text { profissio } \\
\text { nais de } \\
\text { saúde }\end{array}$ & Não relata & $\begin{array}{l}\text { Apesar dos profissionais de enfermagem } \\
\text { serem os mais habituados com a } \\
\text { administração de medicamentos e/ou } \\
\text { terapias de hidratação, estudos } \\
\text { evidenciam que a adesão dos } \\
\text { profissionais de saúde acerca da técnica } \\
\text { de hipodermóclise ainda é restrita. }\end{array}$ \\
\hline $\begin{array}{l}\text { Educação permanente } \\
\text { sobre hipodermóclise } \\
\text { com uma equipe de } \\
\text { enfermagem de uma } \\
\text { unidade de internação } \\
\text { cirúrgica }\end{array}$ & $\begin{array}{l}\text { Relatar a experiência de } \\
\text { uma atividade de educação } \\
\text { permanente sobre } \\
\text { hipodermóclise realizada } \\
\text { com profissionais de } \\
\text { enfermagem no contexto } \\
\text { hospitalar }\end{array}$ & Não relata & $\begin{array}{l}16 \\
\text { técnicos } \\
\text { de } \\
\text { enfermag } \\
\text { em e } 6 \\
\text { auxiliares } \\
\text { de } \\
\text { enfermag } \\
\text { em }\end{array}$ & Não relata & $\begin{array}{l}\text { Muitos profissionais médicos e de } \\
\text { enfermagem têm pouco conhecimento } \\
\text { sobre essa técnica e necessitam de } \\
\text { capacitações e programas de } \\
\text { treinamentos específicos para } \\
\text { implementá-la. }\end{array}$ \\
\hline $\begin{array}{l}\text { Hypodermoclysis with } \\
\text { older adults }\end{array}$ & $\begin{array}{l}\text { Abordar acerca da } \\
\text { hipodermóclise. }\end{array}$ & Desidratação & $\begin{array}{ll}\text { Não } & \text { se } \\
\text { aplica }\end{array}$ & $\begin{array}{l}\text { Sangramento } \\
\text { local, edema, } \\
\text { calor, eritema, } \\
\text { equimoses, dor, } \\
\begin{array}{l}\text { abscesso, } \\
\text { infecção }\end{array}\end{array}$ & $\begin{array}{l}\text { O artigo apresenta os benefícios da } \\
\text { hipodermóclises em pacientes mais } \\
\text { velhos com desidratação }\end{array}$ \\
\hline $\begin{array}{lr}\text { Hipodermóclise: } & \text { o } \\
\text { conhecimento } & \text { do } \\
\text { enfermeiro } & \text { em } \\
\text { unidade de internação }\end{array}$ & $\begin{array}{l}\text { Avaliar o conhecimento } \\
\text { dos enfermeiros da unidade } \\
\text { de internação em relação à } \\
\text { hipodermóclise }\end{array}$ & Não relata & $\begin{array}{l}7 \\
\text { Enfermei } \\
\text { ros }\end{array}$ & Não relata & $\begin{array}{l}\text { Predomina o desconhecimento da } \\
\text { hipodermóclise entre os enfermeiros da } \\
\text { unidade de internação, fazendo-se } \\
\text { necessária a abordagem do tema em } \\
\text { âmbito hospitalar e no meio acadêmico. }\end{array}$ \\
\hline $\begin{array}{l}\text { Hipodermóclise em } \\
\text { pacientes oncológicos } \\
\text { em cuidados paliativos }\end{array}$ & $\begin{array}{l}\text { Descrever a experiência da } \\
\text { utilização da } \\
\text { hipodermóclise } \\
\text { pacientes sob cuidados } \\
\text { paliativos e tratamento da } \\
\text { dor, em hospital de } \\
\text { referência em oncologia do } \\
\text { Paraná }\end{array}$ & $\begin{array}{l}\text { Analgesia em fase final } \\
\text { de vida }\end{array}$ & $\begin{array}{l}16 \\
\text { participa } \\
\text { ntes com } \\
\text { diagnósti } \\
\text { co } \\
\text { oncológic } \\
\text { o }\end{array}$ & $\begin{array}{l}\text { Leve irritação no } \\
\text { local de punção e } \\
\text { hematoma }\end{array}$ & $\begin{array}{l}\text { Demonstrou que a hipodermóclise é } \\
\text { uma técnica segura, confiável, de fácil } \\
\text { manipulação e com o mínimo de } \\
\text { desconforto para o paciente. }\end{array}$ \\
\hline $\begin{array}{l}\text { A Prospective Study } \\
\text { of Hypodermoclysis } \\
\text { Performed by } \\
\text { Caregivers in the } \\
\text { Home Setting }\end{array}$ & 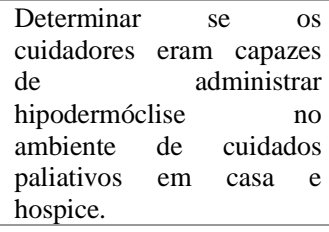 & Reidratação & $\begin{array}{l}21 \\
\text { cuidadore } \\
\mathrm{s}\end{array}$ & Vermelhidão & $\begin{array}{l}\text { Sugere que a hidratação subcutânea } \\
\text { pode ser administrada por cuidadores } \\
\text { em casa com carga mínima, } \\
\text { equipamento e suporte técnico. }\end{array}$ \\
\hline $\begin{array}{lr}\text { Hipodermóclise } & \text { para } \\
\text { tratamentos } & \text { não } \\
\text { convencionais } & \text { em } \\
\text { pediatria: } & \text { Revisão } \\
\text { integrativa } & \\
\end{array}$ & $\begin{array}{l}\text { Descrever o uso da } \\
\text { hipodermóclise } \\
\text { tratamentos pediátricos não } \\
\text { convencionais }\end{array}$ & $\begin{array}{ll}\text { Terapia medicamentosa } & \\
\text { (diabetes e alergias } \\
\text { infantis), nutrição e e } \\
\text { hidratação }\end{array}$ & $\begin{array}{ll}\text { Não se } \\
\text { aplica }\end{array}$ & Dor e ansiedade & $\begin{array}{l}\text { Limitado a elaboração de tipos de } \\
\text { estudos experimentais que elucidem a } \\
\text { efetividade da hipodermóclise. }\end{array}$ \\
\hline $\begin{array}{l}\text { Hipodermoclisis en } \\
\text { pacientes con cáncer } \\
\text { terminal }\end{array}$ & $\begin{array}{l}\text { Saber se é viável } \\
\text { administrar hidratação } \\
\text { subcutânea em pacientes } \\
\text { com doenças terminais do } \\
\text { câncer, as características da } \\
\text { técnica e as dificuldades } \\
\text { que possam surgir durante } \\
\text { a sua aplicação }\end{array}$ & $\begin{array}{lr}\text { Hidratação } & \text { para } \\
\text { pacientes } & \text { com } \\
\text { incapacidade de } & \text { ingerir } \\
\text { líquidos por V.O. } & \end{array}$ & $\begin{array}{l}10 \\
\text { pacientes } \\
\text { com } \\
\text { câncer } \\
\text { avançado }\end{array}$ & $\begin{array}{lr}\text { Absorção } \\
\text { insuficiente com } \\
\text { acúmulo local } \\
\text { excessivo de } \\
\text { fluido no espaço } \\
\text { celular subcutâneo } \\
\text { e desconforto no } \\
\text { local da punção }\end{array}$ & $\begin{array}{l}\text { A utilização da via subcutânea como } \\
\text { alternativa à via intravenosa para } \\
\text { hidratação de pacientes em estágio final } \\
\text { de vida é simples e tem um baixo } \\
\text { incidência de efeitos adversos e } \\
\text { complicações }\end{array}$ \\
\hline $\begin{array}{l}\text { Medicamentos } \\
\text { passíveis de infusão } \\
\text { por hipodermóclise }\end{array}$ & $\begin{array}{l}\text { Analisar criticamente as } \\
\text { evidências científicas sobre } \\
\text { a infusão de medicações } \\
\text { com indicação de uso por } \\
\text { hipodermóclise. }\end{array}$ & $\begin{array}{l}\text { Analgésicos, fluídos de } \\
\text { reidratação, anti } \\
\text { eméticos, etc }\end{array}$ & 6 artigos & $\begin{array}{l}\text { Edema } \\
\text { irritabilidade local } \\
\text { (a depender a } \\
\text { medicação) }\end{array}$ & $\begin{array}{l}\text { Apresenta uma lista de medicamentos, } \\
\text { indicação, posologia, diluição, tempo de } \\
\text { infusão e observações de cada } \\
\text { medicamento que pode ser administrado } \\
\text { por via hipodermóclise. }\end{array}$ \\
\hline $\begin{array}{ll}\text { Use } & \text { of } \\
\text { hypodermoclysis } & \text { to } \\
\text { manage dehydration } & \end{array}$ & $\begin{array}{l}\text { Realizar um breve relato } \\
\text { sobre o uso da } \\
\text { hipodermóclise em idosos }\end{array}$ & Reidratação & $\begin{array}{ll}\text { Não } & \text { se } \\
\text { aplica } & \end{array}$ & Dor e edema local & $\begin{array}{l}\text { Apresenta a hipodermóclise como } \\
\text { alternativa fácil, segura e econômica, } \\
\text { podendo ser realizada em ambiente } \\
\text { domiciliar, hospice e hospitalar }\end{array}$ \\
\hline $\begin{array}{l}\text { Hypodermoclysis: A } \\
\text { viable rehydration } \\
\text { option? }\end{array}$ & $\begin{array}{l}\text { Fornecer uma visão geral } \\
\text { sobre o que é a } \\
\text { hipodermóclise, para que } \\
\text { pode ser usada e delinear } \\
\text { as vantagens e limitações } \\
\text { de seu uso }\end{array}$ & $\begin{array}{l}\text { Reidratação em crianças } \\
\text { e idosos e pessoas em } \\
\text { cuidados paliativos. }\end{array}$ & $\begin{array}{ll}\text { Não } & \text { se } \\
\text { aplica } & \end{array}$ & $\begin{array}{l}\text { Dor e raramente } \\
\text { celulite }\end{array}$ & $\begin{array}{l}\text { Quando usado para situações } \\
\text { apropriadas por pessoal treinado, a } \\
\text { hipodermóclise oferece muitas } \\
\text { vantagens e poucas desvantagens sobre } \\
\text { outras técnicas de hidratação. }\end{array}$ \\
\hline $\begin{array}{lr}\text { Percepções } & \text { de } \\
\text { cuidadores familiares } \\
\text { sobre o uso da } \\
\text { hipodermóclise } \\
\text { domicílio }\end{array}$ & $\begin{array}{l}\text { Conhecer as percepções de } \\
\text { cuidadores familiares sobre } \\
\text { o uso da hipodermóclise no } \\
\text { domicílio }\end{array}$ & $\begin{array}{l}\text { Reposição de fluídos, } \\
\text { hidratação e como forma } \\
\text { de administração de } \\
\text { medicamentos pela via } \\
\text { subcutânea }\end{array}$ & $\begin{array}{l}10 \\
\text { participa } \\
\text { ntes }\end{array}$ & $\begin{array}{l}\text { Edema, } \\
\text { vermelhidão, dor } \\
\text { e equimose }\end{array}$ & $\begin{array}{l}\text { Os familiares apresentam sentimentos } \\
\text { ambíguos em relação à hipodermóclise. } \\
\text { Sentem medo em assumir as } \\
\text { responsabilidades pelos cuidados com a } \\
\text { hipodermóclise, mas também referem-se }\end{array}$ \\
\hline
\end{tabular}




\begin{tabular}{|c|c|c|c|c|c|}
\hline & & & & & $\begin{array}{l}\text { seguros e satisfeitos com o uso dessa via } \\
\text { de administração de medicamentos pelo } \\
\text { familiar doente no domicílio. }\end{array}$ \\
\hline $\begin{array}{l}\text { Complicações da via } \\
\text { subcutânea na infusão } \\
\text { de medicamentos e } \\
\text { soluções em cuidados } \\
\text { paliativos }\end{array}$ & $\begin{array}{l}\text { Caracterizar } r \text { as } \\
\text { complicações associadas } \\
\text { ao uso da via subcutânea } \\
\text { na infusão r de } \\
\text { medicamentos e soluções } \\
\text { em cuidados paliativos }\end{array}$ & Terapia medicamentosa & $\begin{array}{l}78 \\
\text { pacientes } \\
\text { em } \\
\text { cuidados } \\
\text { paliativos }\end{array}$ & $\begin{array}{l}\text { Hiperemia, } \\
\text { edema, celulite, } \\
\text { sangramento, dor, } \\
\text { abscesso, necrose }\end{array}$ & $\begin{array}{l}\text { O uso da via subcutânea para infusão de } \\
\text { medicamentos e soluções pode ser } \\
\text { considerada uma alternativa de infusão } \\
\text { segura, a qual apresenta risco de } \\
\text { complicações em sua maioria facilmente } \\
\text { reversíveis e com baixo potencial de } \\
\text { ocasionar danos aos pacientes. }\end{array}$ \\
\hline $\begin{array}{ll}\text { Benefícios } & \text { da } \\
\text { Hipodermóclise na } \\
\text { Clínica Paliativa de } \\
\text { Pacientes } \\
\text { com Câncer: Relato de } \\
\text { Caso }\end{array}$ & $\begin{array}{l}\text { Trata-se de dois relatos de } \\
\text { caso de pacientes com } \\
\text { câncer, nos quais a } \\
\text { hipodermóclise }\end{array}$ & Terapia medicamentosa & $\begin{array}{l}2 \\
\text { participa } \\
\text { ntes }\end{array}$ & $\begin{array}{l}\text { Sinais flogísticos } \\
\text { nos locais }\end{array}$ & $\begin{array}{l}\text { Via segura, minimamente invasiva e } \\
\text { eficaz no tratamento sintomático dos } \\
\text { pacientes com câncer em cuidados } \\
\text { paliativos. Recomenda-se que a terapia } \\
\text { subcutânea seja amplamente divulgada } \\
\text { na prática clínica paliativa entre a } \\
\text { equipe multiprofissional. }\end{array}$ \\
\hline $\begin{array}{l}\text { Hipodermoclisis como } \\
\text { alternativa para la } \\
\text { rehidratación en niños }\end{array}$ & $\begin{array}{l}\text { Apresentar } \\
\text { hipodermóclise como uma } \\
\text { via alternativa para a } \\
\text { reidratação em crianças }\end{array}$ & Reidratação em crianças & $\begin{array}{ll}\text { Não } & \text { se } \\
\text { aplica } & \end{array}$ & Edema & $\begin{array}{l}\text { A hipodermóclise se torna uma } \\
\text { alternativa atraente na população } \\
\text { pediátrica graças à técnica simples de } \\
\text { aplicação. }\end{array}$ \\
\hline $\begin{array}{l}\text { Via subcutânea: } \\
\text { segunda opção em } \\
\text { cuidados paliativos }\end{array}$ & $\begin{array}{l}\text { Realizar uma revisão } \\
\text { teórica a respeito do uso da } \\
\text { via subcutânea para } \\
\text { pacientes em cuidados } \\
\text { paliativos. }\end{array}$ & Terapia medicamentosa & $\begin{array}{ll}\text { Não } & \text { se } \\
\text { aplica }\end{array}$ & $\begin{array}{l}\text { Depende da } \\
\text { medicação } \\
\text { utilizada e sua } \\
\text { compatibilidade } \\
\text { com a técnica }\end{array}$ & $\begin{array}{l}\text { Técnica de fácil aplicabilidade e } \\
\text { manutenção em ambiente hospitalar ou } \\
\text { domiciliar; melhora a autonomia e a } \\
\text { qualidade de vida do paciente, sendo } \\
\text { esse o objetivo primordial em cuidados } \\
\text { paliativos }\end{array}$ \\
\hline $\begin{array}{l}\text { Uso de la vía } \\
\text { subcutánea } \\
\text { cuidados del final de } \\
\text { la vida en el Centro } \\
\text { Geriátrico Naval }\end{array}$ & $\begin{array}{l}\text { Descrever o uso da via } \\
\text { subcutânea em cuidados } \\
\text { paliativos do fim da vida }\end{array}$ & $\begin{array}{l}\text { Terapia medicamentosa } \\
\text { em fim de vida }\end{array}$ & $\begin{array}{l}22 \\
\text { pacientes }\end{array}$ & Eritema & $\begin{array}{l}\text { Alternativa eficaz para medicação em } \\
\text { infusão e fluidos em pacientes que } \\
\text { recebem cuidados paliativos. }\end{array}$ \\
\hline $\begin{array}{l}\text { Efeitos Adversos da } \\
\text { Hipodermóclise em } \\
\text { pacientes adultos: } \\
\text { Revisão integrativa }\end{array}$ & \begin{tabular}{lr}
\multicolumn{2}{l}{ Identificar na literatura } \\
artigos relacionados aos \\
efeitos adversos & da \\
hipodermóclise & em \\
pacientes adultos &
\end{tabular} & $\begin{array}{l}\text { Terapia medicamentosa } \\
\text { e hidratação leve a } \\
\text { moderada }\end{array}$ & $\begin{array}{l}\text { Não se } \\
\text { aplica } \\
\text { (Artigos } \\
\text { seleciona } \\
\text { dos } \\
\text { foram } \\
\text { 17) }\end{array}$ & $\begin{array}{l}\text { Dor e edema no } \\
\text { local da punção, } \\
\text { celulite e absorção } \\
\text { insuficiente da } \\
\text { solução rom } \\
\text { acúmulo co de } \\
\text { líquido no local }\end{array}$ & $\begin{array}{l}\text { Os efeitos adversos da hipodermóclise } \\
\text { nos pacientes adultos relatados na } \\
\text { literatura não causaram danos graves aos } \\
\text { pacientes e são, em sua maioria, } \\
\text { mínimos e reversíveis. }\end{array}$ \\
\hline $\begin{array}{lr}\text { Conhecimentos } & \mathrm{e} \\
\text { práticas da } \\
\text { enfermagem } & \text { na } \\
\text { administração } & \text { de } \\
\text { fluidos por } & \text { via } \\
\text { subcutânea } & \end{array}$ & 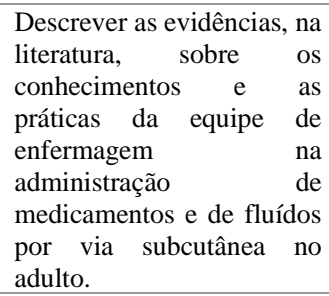 & Terapia medicamentosa & $\begin{array}{l}\text { Não se } \\
\text { aplica }(8 \\
\text { artigos })\end{array}$ & $\begin{array}{l}\text { Raros efeitos } \\
\text { como edema, } \\
\text { irritabilidade e } \\
\text { eritema }\end{array}$ & $\begin{array}{l}\text { Há carência de estudos sobre a } \\
\text { hipodermóclise, sendo necessários mais } \\
\text { investimentos em pesquisas.Mesmo } \\
\text { diante das múltiplas vantagens clínicas } \\
\text { aplicáveis, essa via ainda permanece } \\
\text { subutilizada, havendo necessidade } \\
\text { urgente de mais estudos clínicos para } \\
\text { promover a tomada de decisão e nortear } \\
\text { a prática clínica entre os profissionais. }\end{array}$ \\
\hline $\begin{array}{l}\text { Subcutaneous } \\
\text { hydration by } \\
\text { hypodermoclysis }\end{array}$ & $\begin{array}{l}\text { Esclarecer as indicações, } \\
\text { contra-indicações, } \\
\text { vantagens e desvantagens } \\
\text { desta técnica simples, a fim } \\
\text { de encorajar seu uso em } \\
\text { paciente mais velhos }\end{array}$ & $\begin{array}{lr}\text { Reposição de } & \text { fluidos, } \\
\text { hidratação } & \mathrm{e} \\
\text { administração } & \mathrm{de} \\
\text { medicamentos } & \end{array}$ & $\begin{array}{ll}\text { Não } & \text { se } \\
\text { aplica } & \end{array}$ & $\begin{array}{lr}\text { Dor } & \text { local, } \\
\text { hiperemia. } & \text { Raros } \\
\text { episódios } & \text { de } \\
\text { hiponatremia. }\end{array}$ & $\begin{array}{l}\text { A hidratação subcutânea, ou } \\
\text { hipodermóclise (HDC), é uma técnica } \\
\text { muito simples que deve ser praticada } \\
\text { mais frequentemente por médicos e } \\
\text { enfermeiras que cuidam de pacientes } \\
\text { mais velhos. Envolve menos } \\
\text { desconforto, gera menos custo e tem } \\
\text { menos efeitos adversos do que a via } \\
\text { intravenosa e pode reduzir a necessidade } \\
\text { de admissão ao departamento de } \\
\text { emergência. }\end{array}$ \\
\hline
\end{tabular}

Fonte: Dados da Pesquisa (2020).

\section{Discussão}

Através deste estudo e seus resultados, foi possível observar que os profissionais de saúde que mais publicaram acerca do tema foram os enfermeiros. Isso se deve ao fato de que esses profissionais são os que mais estão habituados com a administração de medicamentos e terapias de hidratação. Entretanto, médicos e farmacêuticos também apresentam interesse pelo tema, uma vez que é necessário que a diluição e posologia dos medicamentos sejam adequadas para a utilização na via subcutânea (Takaki \& Klein, 2010; Gomes et al., 2019). 
A grande maioria dos estudos apontam a hipodermóclise como uma técnica segura, eficaz, com poucos ou raros efeitos adversos, menos dolorosa que a via endovenosa e de baixo custo, porém, pouco difundida entre os profissionais de saúde. Tal fato pode ser explicado por dois principais motivos: a prática incorreta da técnica na antiguidade e a restrita, ou até mesmo ausente, difusão do conhecimento teórico-prático sobre a hipodermóclise durante a formação acadêmica dos profissionais de saúde, resultando no desconhecimento e não uso da técnica. (Martin, 2010; Lopez \& Reyes-Ortiz, 2010; Smith, 2014; Gomes et al., 2019)

Outro aspecto observado durante a análise da amostra selecionada para esse estudo, foi a tímida, porém, crescente introdução da hipodermóclise na pediatria. Por se tratar de uma técnica que envolve apenas uma punção simples no subcutâneo, reduz o desconforto de repetidas tentativas de puncionar uma rede venosa fragilizada, o que gera mais conforto e menos estresse às crianças, principalmente as menores de um ano de idade. Dessa maneira, a prática vem se tornando uma escolha alternativa na falha da via endovenosa convencional. Todavia, ressalta-se que o uso é citado para casos de desidratação leve a moderada, pois em casos de desidratação grave é necessário uma grande e rápida reposição volêmica. Mesmo apresentando-se uma ferramenta valiosa para a pediatria, requer mais estudos e pesquisas com metodologias de alto nível de evidência e qualidade metodológica, visando respaldar e disseminar o conhecimento e o uso da hipodermóclise na pediatria. (D’Amato et al., 2014; Saganski \&, Freire, 2019; Saganski et al., 2019).

A desidratação é um diagnóstico comum aos idosos, em especial, os mais frágeis, devido às mudanças fisiológicas e comorbidades próprias da idade. Nesse contexto, a hipodermóclise tem sido utilizada com grandes êxitos, apresentando excelentes resultados no controle de desidratação leve a moderada, maior aceitabilidade pelo paciente, maior durabilidade da punção, baixo custo, economia de tempo e facilidade na execução da técnica pelos profissionais da equipe (Scales 2011; Broadhurst, 2012; Garrett, 2013; Russell, 2018).

Apesar do interesse e uso da via subcutânea por outras especialidades, a hipodermóclise tem sido empregada, majoritariamente, aos pacientes oncológicos adultos e em cuidados paliativos. Esse perfil de paciente costuma apresentar, desidratação, rede venosa periférica frágil, de difícil acesso, náuseas, vômitos e dificuldades para deglutir, impossibilitando as vias endovenosa e oral. Assim, a via subcutânea se torna uma opção de escolha, pois além de ser uma técnica simples, apresenta menor grau de limitação devido as diversas opções de sítios de punção, baixo risco de infecção, ser uma via segura para administração de opioides, antieméticos, quimioterápicos, dentre outros, sem risco de trombose, menor risco do acesso ficar disfuncional e maior aceitabilidade do paciente e família (Pontalti et al., 2012; Justino et al., 2013; Bruno, 2015; Gomes et al., 2019).

Como a técnica da hipodermóclise é simples, de fácil manuseio e segura, também tem alcançado lugar de destaque na atenção domiciliar aos pacientes em cuidados paliativos. Com a devida capacitação, os cuidadores domiciliares podem administrar medicações e fluidos de hidratação através da via subcutânea, contribuindo para o conforto e bem estar deste paciente, uma vez que este não precisa ser constantemente hospitalizado para controle de sintomas como náuseas, vômitos e desidratação (Martins et al., 2019; Vidal et al., 2016).

Quanto aos efeitos adversos da hipodermóclise, a seleção desse estudo apontou dor, hiperemia, eritema e edema no local da punção como os principais relatos. Tais sinais, são de repercussão mínima ao paciente e reversíveis, sendo controlados com massagem, troca do local de punção e diminuição da velocidade de infusão. Entretanto, em raros casos, efeitos mais graves como celulite e necrose tecidual local foram relatados e associados a prática errônea da técnica, administração de medicamentos em diluição não apropriada ou medicações que não possuíam indicação para uso na via subcutânea. Por conseguinte, para esses efeitos preconiza-se o uso de antibióticos, compressas frias e acompanhamento diário do local de punção. Além disso, recomenda-se a capacitação dos profissionais que irão realizar a técnica e a constante atualização sobre a mesma, destacando a importância da escolha em local apropriado para punção (com maior quantidade de tecido subcutâneo) e 
não exceder o tempo de permanência da agulha no local de punção, que varia entre 48 e 96 horas (Lopez \& Reyes-Ortiz, 2010; Rodrigues et al., 2016; Guedes et al., 2019).

\section{Conclusão}

Os resultados apreciados nesta scoping review oferecem evidências do protagonismo da enfermagem na realização da hipodermóclise e do avanço do conhecimento da referida área. Além de compilar informações que podem direcionar os profissionais de saúde para a utilização da referida técnica, com vistas a garantir o sucesso da terapia e a segurança do paciente.

Os estudos submetidos à análise enfocam vários ambientes de atuação desses profissionais, apresentando desafios e contribuições que perpassam a educação em saúde, o gerenciamento de equipes, além do gerenciamento de possíveis eventos adversos relacionados à administração por tal via. Apesar da sistematização empregada na busca e na seleção dos artigos incluídos nesta revisão, reconhece-se o desafio de alcançar a totalidade dos estudos produzidos.

Ademais, espera-se que as lacunas aqui ressaltadas motivem o desenvolvimento de novos estudos com maior rigor metodológico, visando a produção de evidências científicas confiáveis sobre a utilização da técnica da hipordermóclise por profissionais de saúde.

\section{Referências}

Broadhurst, D. (2012). Hydrating Your Patient the Easy Way: Hypodermoclysis. Vascular Access, 7-19. http://search.ebscohost.com/login.aspx?direct=true \&db=c8h\&AN=104061929\&lang=pt-br\&site=ehost-live

Bruno, V. G. (2015, March). Hipodermóclise: revisão de literatura para auxiliar a prática clínica. Einstein (Sao Paulo), 13, $122-128$. https://doi.org/10.1590/s1679-45082015rw2572

Candon, L. H., Van Toen, J., \& Amirov, C. (2010). A multifaceted intervention to address a case cluster of cellulitis associated with hypodermoclysis in a geriatric complex continuing care unit. Canadian Journal of Infection Control, 25, 101-106.

http://search.ebscohost.com/login.aspx?direct=true \&db=c8h\&AN=105074980\&lang=pt-br\&site=ehost-live

D’aquino, M. O., Souza, R. M. (2012) Hipodermóclise ou via Subcutânea. Revista do Hospital Universitário Pedro Ernesto, UERJ. https://www.epublicacoes.uerj.br/index.php/revistahupe/article/view/8948/6841

D’Amato G, M., Escobar V, A., Fernández L, M., \& Irazuzta, J. E. (2014). Hipodermoclisis como alternativa para la rehidratación en niños. Med. U.P.B, 33, 138-144. https://pesquisa.bvsalud.org/portal/resource/pt/biblio-836899

Garrett, D. (2013). Use of hypodermoclysis to manage dehydration: Practice question. Nursing Older People, 25(4), 12-12. doi: 10.7748 / nop2013.05.25.4.12.s18.

Gomes, N. S., Silva, A. M. B. d., Zagol, L. B. (2017). Conhecimentos e práticas da enfermagem na administração de fluidos por via subcutânea. Revista Brasileira de Enfermagem, 70, 1096-105. https://doi.org/http://dx.doi.org/10.1590/0034-7167-2016-0424

Gomes, N. S., Oliveira, T. R., Silva, A. M. B., \& Barichello, E. (2019). Validação de instrumento para avaliação do conhecimento profissional acerca da hipodermóclise. Revista de Enfermagem e Atenção à Saúde - Reas, 8, 103-117. https://doi.org/10.18554/reas.v8i1.3432

Guedes, N. d. A. B., Melo, L. S. d., Santos, F. B. O., \& Barbosa, J. A. . G. (2019). Complicações da via subcutânea na infusão de medicamentos e soluções em cuidados paliativos. Rev Rene, 20, e40933. https://doi.org/10.15253/2175-6783.20192040933

Hernández Perera, Abel, Hall Smith, Charles, \& Hernández Perera, Aliana. (2011). Hipodermoclisis en pacientes con cáncer terminal. Revista Cubana de Medicina, 50(2), 150-156. Recuperado en 14 de julio de 2021, de http://scielo.sld.cu/scielo.php?script=sci_arttext\&pid=S0034-

$75232011000200005 \& \operatorname{lng}=$ es\&tlng=es.

Humphrey, P. (2011). I.V. ROUNDS. Hypodermoclysis: An alternative to I.V. infusion therapy. Nursing, 41, 16-17. https://doi.org/10.1097/01.NURSE.0000405126.91849.e3

Justino, E. T., Tuoto, F. S., Kalinke, L. P., \& Mantovani, M. d. F. (2013). Hipodermóclise em pacientes oncológicos sob cuidados paliativos. Cogitare Enfermagem, 18, 84-89. http://search.ebscohost.com/login.aspx?direct=true\&db=c8h\&AN=87636947\&lang=pt-br\&site=ehost-live

Levac, D., Colquhoun, H. \& O’Brien, K. K. (2010). Scoping studies: advancing the methodology. Implement Sci, 5, (1):69. Disponível em: <https://implementationscience.biomedcentral.com/articles/10.1186/1748-5908-5-69>

1Lopez, J. H., \& Reyes-Ortiz, C. A. (2010). Subcutaneous hydration by hypodermoclysis. Reviews in Clinical Gerontology, $20,105-113$. https://doi.org/10.1017/S0959259810000109

Martin, C. M. (2010). Hypodermoclysis: renewed interest in an old technique. Consultant Pharmacist, 25, 204-212. https://doi.org/10.4140/TCP.n.2010.204 
Martins, S. B., Cordeiro, F. R., Zillmer, J. G. V., Arrieira, I. C. O., Oliveira, A. T., \& Santos, C. d. (2019). Percepções de cuidadores familiares sobre o uso da hipodermóclise no domicílio. Revista Enfermería Actual, 38. https://doi.org/DOI 10.15517/revenf.v0i38.38509

Nunes, P. M. d. S. A., \& Souza, R. C. S. (2016). Efeitos adversos da hipodermóclise em pacientes adultos: revisão integrativa. REME - Revista Mineira de Enfermagem, 20, e951. https://doi.org/http://www.dx.doi.org/10.5935/1415-2762.20160020

Peters, M. D. J, Godfrey, C., McInerney, P., Munn, Z., Tricco, A. C. \& Khalil, H. (2020). Capítulo 11: Revisões do escopo (versão 2020). In: Aromataris E, Munn Z (Editores). JBI Manual for Evidence Synthesis , JBI. Disponível em https://synthesismanual.jbi.global . https://doi.org/10.46658/JBIMES-20-12

Pino, C., Parodi , J., Gonzáles, V., \& Morante, R. (2011). Uso de la vía subcutánea en cuidados del final de la vida en el Centro Geriátrico Naval. Revista Horizonte Médico (Lima), 11, 36-39. https://doi.org/10.24265/horizmed

ontalti, G., Riboldi, C. d. O., Gioda, R. S., Echer, I. C., Franzoi, M. A., \& Wegner, W. (2017). Benefícios da Hipodermóclise na Clínica Paliativa de Pacientes com Câncer: Relato de Caso. Revista Brasileira de Cancerologia, 62, 247-252. https://doi.org/10.32635/2176-9745.RBC.2016v62n3.222

Pontalti, G., Rodrigues, E. S. A., Firmino, F., Fábris, M., Stein, M. R., \& Longaray, V. K. (2012). Via subcutânea: segunda opção em cuidados paliativos. Clinical \& Biomedical Research, 32(2). https://seer.ufrgs.br/hcpa/article/view/26270

Rodrigues, F. S., Pedra, M. C., Santos, J. L. G. d., Rumor, P. C. F., Girondi, J. B. R., \& Oliveira, M. C. d. (2016). Educação permanente sobre hipodermóclise com a equipe de enfermagem de uma unidade de internação cirúrgica. Revista de Enfermagem UFPE, 1562-1570. https://doi.org/10.5205/reuol.7057-60979-3SM-1.1003sup201625

Russell, S. (2018, April). Hypodermic clysis: A viable rehydration option? Geriatric Nursing, 39, 247-249. https://doi.org/10.1016/j.gerinurse.2018.02.007

Saganski, G. F., \& Freire, M. H. d. S. (2019). Segurança e eficácia da hipodermóclise em comparação com a infusão de fluido intravenoso para reidratar crianças com desidratação leve a moderada: um protocolo de revisão sistemática. JBI Database of Systematic Reviews \& Implementation Reports, 17, 12701276. https://doi.org/10.11124/JBISRIR-2017-003696

Saganski, G. F., Freire, M. H. d. S., Peres, A. L., Gusso, A. . K., Moraes, S. d. R. L., \& Migoto, M. T. (2019). Hipodermóclise para tratamentos não convencionais em pediatria: revisão integrativa. Cogitare Enfermagem, 24, e61546. https://doi.org/http://dx.doi.org/10.5380/ce.v24i0.61546

Scales, K. (2011). Use of hypodermoclysis to manage dehydration. Nursing Older People, 23, 16-22. https://doi.org/10.7748/nop2011.06.23.5.16.c8528

Smith, L. S. (2014). CLINICAL QUERIES. Hypodermoclysis with older adults. Nursing, 44, 12-12. https://doi.org/10.1097/01.NURSE.0000456386.55897.ff

Takaki, C. Y. I., \& Klein, G. d. F. S. (2010). Hipodermóclise: o conhecimento do enfermeiro em unidade de internação. ConScientiae Saude, 9, $486-496$. https://doi.org/http://search.ebscohost.com/login.aspx?direct=true\&db=c8h\&AN=104941170\&lang=pt-br\&site=ehost-live

Tricco, A. C, et al. PRISMA extension for scoping reviews (PRISMA-ScR): checklist and explanation. Ann Intern Med. 2018;169(7):467-73. DOI: $10.7326 / \mathrm{M} 18-0850$

Vidal, M., Hui, D., Williams, J., \& Bruera, E. (2016). A Prospective Study of Hypodermoclysis Performed by Caregivers in the Home Setting. Journal of Pain \& Symptom Management, 52, 570-574.e9. https://doi.org/10.1016/j.jpainsymman.2016.04.009

Quaglio, R. de C., Varallo, F. R., Lima, N. K. da C., Junqueira, A. F., Ianhez Júnior, E., Matumoto, S., \& Fortuna, C. M. (2018). Medicamentos passíveis de infusão por hipodermóclise. Medicina (Ribeirão Preto), 51(1), 55-68. https://doi.org/10.11606/issn.2176-7262.v51i1p55-68

Zironde, E. S., Marzenini, N. L. \& Soler, V. M. (2014). Hipodermóclise: redescoberta da via subcutânea no tratamento de indivíduos vulneráveis. Cuidarte Enfermagem., v. 8, n. 1, p. 55-61, janeiro-junho. 